CERN-TH 6638/92

CPT-92/PE.2799

ENSLAPP-A-398/92

\title{
Invariants of Three-Manifolds from Finite Group Cohomology*
}

\author{
Daniel Altschuler \\ CERN, Theory Division \\ CH-1211 Genève 23, Switzerland \\ and \\ Antoine Coste \\ Centre de Physique Théorique \\ CNRS Luminy case 907 \\ F-13288 Marseille cedex 9, France
}

\begin{abstract}
We compare on lens spaces the values of two topological invariants of three-manifolds, both built from a finite group $G$ and a 3-cocycle $\omega$, which we conjectured to be equal up to a normalization. The first invariant is defined by triangulation, it is the partition function of the Dijkgraaf-Witten topological field theory, and the second one by surgery, using a quasi-Hopf algebra. When $G$ is a cyclic group, we show that the first invariant reduces to a Gauss sum. Some identities satisfied by 3-cocycles are derived in an appendix.
\end{abstract}

CERN-TH 6638/92

September 1992

*To appear in the proceedings of the Karpacz Winter School of Theoretical Physics, February 1992 


\section{Introduction}

In this paper we present some detailed computations of partition functions in what is one of the simplest examples of 3-dimensional topological field theory, which was defined by Dijkgraaf and Witten [1]. It is an analog of the more well-known Chern-Simons theory [2] in the case of finite gauge groups. To each compact, closed, oriented 3-manifold $M$ it associates a complex number $Z(M)$ (the partition function) which is a topological invariant of $M$. This invariant depends only on the finite group $G$, and the cohomology class of a 3 -cocycle $\omega$ with values in $U(1)$. In the same way as the Chern-Simons theory is related to the two-dimensional WZW model, the Dijkgraaf-Witten theory is related to another class of conformal field theories, the so-called holomorphic orbifolds [3].

Following [1] we will give an elementary combinatorial definition of $Z(M)$ in terms of a triangulation. A more abstract definition involving Eilenberg-Mac Lane spaces $K(G, 1)$ was also given by the same authors, but we shall not use it here. Recently in [4] we took another approach, starting from a surgery presentation of $M$ to define a 3-manifold invariant $\mathcal{F}(M)$ depending on the same data $G, \omega$. More precisely, extending the Reshetikhin-Turaev construction $[5,6]$ to the case of quasi-Hopf algebras [7], we considered invariants of links coloured by the regular representation of a finite-dimensional quasitriangular quasi-Hopf algebra $D^{\omega}(G)$, and then proved that they are conserved under Kirby moves [8].

We conjectured that $Z(M)$ and $\mathcal{F}(M)$ are equal (up to a normalization) and checked it in a few cases. The algebra $D^{\omega}(G)$ was defined previously in [9], where it was shown that the structure constants of its ring of representations agree with the fusion rules of [3].

So far a proof of the above conjecture is still lacking. The main difficulty is to relate the two presentations of $M$, by triangulation and surgery. In the sequel we first give the definition and some general properties of the partition function $Z(M)$. To illustrate these, we present detailed computations of the invariants of lens spaces $Z(L(p, q))$, and then give a general formula for the surgery invariants $\mathcal{F}(L(p, q))$.

Before we start to study the invariants, let us recall the definition of a 3-cocycle. Let $G$ be a finite group. We denote its order by $|G|$, and the unit element by $e$. A 3-cocycle is a map $G \times G \times G \rightarrow U(1)$ satisfying

$$
\omega\left(g_{1}, g_{2}, g_{3}\right) \omega\left(g_{1}, g_{2} g_{3}, g_{4}\right) \omega\left(g_{2}, g_{3}, g_{4}\right)=\omega\left(g_{1} g_{2}, g_{3}, g_{4}\right) \omega\left(g_{1}, g_{2}, g_{3} g_{4}\right)
$$

for all $g_{i} \in G, i=1, \ldots, 4$. We also assume that $\omega$ is normalized, i.e. $\omega\left(g_{1}, g_{2}, g_{3}\right)=1$ if at least one of the arguments $g_{i}=e$. Later we will use several identities which are consequences of (1.1). They are collected in the appendix.

One knows $[1,4]$ that the invariants we consider below depend only on the cohomology 
class of $\omega$ : they are invariant under $\omega \mapsto \omega \delta \eta$. Here $\eta$ is a normalized 2-cochain, i.e. a map $G \times G \rightarrow U(1)$ such that $\eta(x, y)=1$ if $x$ or $y=e$, and

$$
\delta \eta(x, y, z)=\eta(y, z) \eta(x y, z)^{-1} \eta(x, y z) \eta(x, y)^{-1} .
$$

Sometimes we will use the following definitions: $\left\{C_{A}\right\}_{A=1, \ldots, P}$ will denote the set of conjugacy classes of $G,\left|C_{A}\right|$ the number of elements in $C_{A}$ and $\left\{g_{A}\right\}_{A=1, \ldots, P}$ a system of representatives of these classes. Also, in this paper the notation $(m, n)$ stands for the greatest common divisor of two integers $m$ and $n$.

\section{Dijkgraaf-Witten invariants}

Let $M$ be a compact oriented 3-manifold without boundary, and $T$ be a triangulation. Denote by $T^{j}$ the set of $j$-simplices of $T$. An oriented tetrahedron $t \in T^{3}$ is given by an ordered 4-tuple of vertices, $t=(a b c d), a, b, c, d \in T^{0}$. Similarly a triangle is given by an ordered triple $(a b c) \in T^{2}$, and an edge by a couple $(a b) \in T^{1}$.

The definition of the partition function is in many respects analogous to lattice gauge theory. In particular, the dynamical variables live on the edges. Thus we define a state to be a map $\rho: T^{1} \rightarrow G,(a b) \mapsto g_{a b}$, such that for any triangle $(a b c) \in T^{2}$, the flatness condition $g_{a b} g_{b c} g_{c a}=e$ is satisfied. We also assign $g_{b a}=g_{a b}^{-1}$ to the edge $(b a)$ with the opposite orientation.

The values of $\rho$ on the edges of a tetrahedron $t=(a b c d)$ are completely determined once $g_{a b}, g_{b c}$ and $g_{c d}$ are given. We define the weight of $t$ by $W_{t}=\omega\left(g_{a b}, g_{b c}, g_{c d}\right)$, and the weight of a state $\rho$ by

$$
W(\rho)=\prod_{t \in T^{3}} W_{t}
$$

The partition function is a sum over a certain set of states, which must be defined carefully in order to avoid overcounting due to gauge invariance of $W$, as we now explain. Any function $\Omega: T^{0} \rightarrow G, a \mapsto \Omega_{a}$, gives rise to a new state $\rho^{\Omega}:(a b) \mapsto \Omega_{a} g_{a b} \Omega_{b}^{-1}$. It can be shown that (1.1) implies that $W$ is gauge invariant, $W\left(\rho^{\Omega}\right)=W(\rho)$. The group $\mathcal{G}$ of gauge transformations acts on the set of all states $\mathcal{M}$. Consider the subgroup $\mathcal{G}_{0}$ consisting of those $\Omega$ which leave a distinguished point $* \in T^{0}$ fixed: $\Omega_{*}=e$. Then the Dijkgraaf-Witten partition function is:

$$
Z(M)=|G|^{-1} \sum_{\rho \in \mathcal{M} / \mathcal{G}_{0}} W(\rho)
$$


One proves $[1,10]$ that $Z(M)$ is independent of the triangulation $T$, so that it is a topological invariant of oriented manifolds. Furthermore, $Z(M) / Z\left(S^{3}\right)$ is multiplicative under connected sum.

Our conjecture is that $Z(M) / Z\left(S^{3}\right)$ is equal to the surgery invariant $\mathcal{F}(M)$ described in [4]. In the simpler case of a trivial cocycle this has been proven by Hennings [13].

Note that if $M$ is connected, then there are no gauge transformations $\Omega \in \mathcal{G}_{0}$ such that $\rho^{\Omega}=\rho$ other than the identity. This is because for any vertex $a \in T^{0}$, there exists a path $\gamma_{a}$ going from $*$ to $a$ consisting of edges of $T^{1}$. Then for any vertex $a \in T^{0}$, the ordered product $g\left(\gamma_{a}\right)$ of elements $g_{x y}$ along this path transforms into $g^{\Omega}\left(\gamma_{a}\right)=g\left(\gamma_{a}\right) \Omega_{a}^{-1}=g\left(\gamma_{a}\right)$, hence $\Omega_{a}=e$. This implies that the orbit of any state $\rho$ has $|G|^{v-1}$ elements, where $v$ is the number of vertices, and

$$
Z(M)=|G|^{-v} \sum_{\rho \in \mathcal{M}} W(\rho)
$$

Proposition There is a one-to-one correspondence between $\mathcal{M} / \mathcal{G}_{0}$ and $\operatorname{Hom}\left(\pi_{1}(M), G\right)$. (Here by $\pi_{1}(M)$ we really mean the fundamental group $\pi_{1}(M, *)$ with basepoint $*$.)

Proof. To each state we can associate a homomorphism $\chi: \pi_{1}(M) \rightarrow G$ by taking the ordered products of elements $g_{a b}$ along closed paths based at $*$. This mapping factors through the projection $Q: \mathcal{M} \rightarrow \mathcal{M} / \mathcal{G}_{0}$. Call $P$ the quotient map $\mathcal{M} / \mathcal{G}_{0} \rightarrow \operatorname{Hom}\left(\pi_{1}(M), G\right)$. Conversely, for any such morphism $\chi$ we can construct a state $\rho^{\prime}$ in the following way. First we choose for each $a \in T^{0}$ a path $\gamma_{a}$ from $*$ to $a$. Then if $(a b) \in T^{1}$ we set $g_{a b}^{\prime}=\chi\left(\left[\gamma_{a b}\right]\right)$, where $\gamma_{a b}$ is the closed path obtained by joining $\gamma_{a}$, the edge $(a b)$ and the path $\gamma_{b}$ taken with the opposite orientation, and $\left[\gamma_{a b}\right]$ is its equivalence class in $\pi_{1}(M)$. Call $X$ this mapping $X: \operatorname{Hom}\left(\pi_{1}(M), G\right) \rightarrow \mathcal{M}, \chi \mapsto \rho^{\prime}$. Let us now prove that:

$$
\begin{aligned}
P \circ Q \circ X & =\operatorname{Id}_{\operatorname{Hom}\left(\pi_{1}(M), G\right)} \\
Q \circ X \circ P & =\operatorname{Id}_{\mathcal{M} / \mathcal{G}_{0}} .
\end{aligned}
$$

First notice that if $(* a) \in T^{1}, g_{* a}^{\prime}$, which is the image by $\chi$ of the trivial homotopy class, equals $e$. Along a path $\gamma: * \rightarrow a_{1} \rightarrow \cdots \rightarrow a_{n} \rightarrow *$, with $\left(* a_{1}\right),\left(a_{i} a_{i+1}\right),\left(a_{n} *\right) \in T^{1}$ one can express the ordered product $g^{\prime}(\gamma)$ as:

$$
\begin{aligned}
g^{\prime}(\gamma) & =g_{* a_{1}}^{\prime} g_{a_{1} a_{2}}^{\prime} \ldots g_{a_{n} *}^{\prime}=\chi\left(\left[\gamma_{a_{1} a_{2}}\right]\right) \ldots \chi\left(\left[\gamma_{a_{n-1} a_{n}}\right]\right) \\
& =\chi\left(\left[\gamma_{a_{1} a_{2}} \ldots \gamma_{a_{n-1} a_{n}}\right]\right)=\chi([\gamma])
\end{aligned}
$$

proving the first equality. For the second one, consider $\rho=\left\{g_{a b}\right\}$, a representative of a class modulo $\mathcal{G}_{0}$, build $\chi=P([\rho])$ and $\rho^{\prime}=\left\{g_{a b}^{\prime}\right\}=X(\chi)$. We now define $\Omega_{a}$ as the ordered product of the elements $g_{x y}$ from $*$ to $a$ along the chosen path $\gamma_{a}$, and also set $\Omega_{*}=e$. Then 
by definition $g_{a b}^{\prime}=\Omega_{a} g_{a b} \Omega_{b}^{-1}$ for every $(a b) \in T^{1}$ proving that $X(\chi)$ and $\rho$ are in the same class modulo $\mathcal{G}_{0}$.

An immediate consequence is that $Z\left(S^{3}\right)=|G|^{-1}$, because $\pi_{1}\left(S^{3}\right)=1$.

Before discussing the examples $M=L(p, q)$, we would like to sketch another description of states in terms of fiber bundles, which is used in $[1,11]$. Consider principal fiber bundles $p: E \rightarrow M$ with structure group contained in $G$, or $G$-bundles for short. Two $G$-bundles $p: E \rightarrow M$ and $p^{\prime}: E^{\prime} \rightarrow M$ are called equivalent if there is a homeomorphism $h: E \rightarrow E^{\prime}$ such that $p^{\prime} \circ h=p$. Now from the definition it is clear that each state $\rho$ defines a $G$ bundle over $M$ : just choose a suitable open neighborhood $U_{a}$ of each $a \in T^{0}$ and interpret the $g_{a b}$ as transition functions in $U_{a} \cap U_{b}$. Conversely, each $G$-bundle with the coordinate neighborhoods $U_{a}$ determines a state $\rho \in \mathcal{M}$.

A theorem of [12] implies that two states $\rho$ and $\rho^{\prime}$ define equivalent bundles if and only if $\rho^{\prime}=\rho^{\Omega}$ for some $\Omega \in \mathcal{G}$. Hence we have a bijection between $\mathcal{M} / \mathcal{G}$ and the equivalence classes of $G$-bundles. On the other hand, to each $G$-bundle we can associate a homomorphism $\chi: \pi_{1}(M) \rightarrow G$ by lifting the closed paths in $M$ to the total space. According to another theorem of [12] two $G$-bundles are equivalent if and only if the corresponding homomorphisms $\chi$ and $\chi^{\prime}$ are equal up to a conjugation: $\chi^{\prime}(\gamma)=g \chi(\gamma) g^{-1}$, for some $g \in G$. Thus we get another bijection, between equivalence classes of $G$-bundles and $\operatorname{Hom}\left(\pi_{1}(M), G\right) / G$.

\section{Dijkgraaf-Witten invariants for lens spaces}

Lens spaces $L(p, q), 0<q<p,(p, q)=1$, are oriented compact 3-manifolds, which result from identifying on the sphere $S^{3}=\left\{\left.(x, y) \in \mathbb{C}^{2}|| x\right|^{2}+|y|^{2}=1\right\}$ the points which belong to the same orbit under the action of $\mathbb{Z}_{p}$ defined by $(x, y) \mapsto\left(w x, w^{q} y\right)$ with $w=\exp (2 i \pi / p)$.

Let us recall some important results about lens spaces [14]. The fundamental group of $L(p, q)$ is $\mathbb{Z}_{p} . L(p, q)$ and $L\left(p, q^{\prime}\right)$, are homeomorphic if and only if:

$$
q= \pm q^{\prime} \quad(\bmod p) \text { or } q q^{\prime}= \pm 1 \quad(\bmod p)
$$

They are of the same homotopy type if and only if there exist an integer $a$ such that:

$$
q q^{\prime}= \pm a^{2} \quad(\bmod p)
$$

In all cases the orientations are preserved if and only if the relevant sign is + . (Two manifolds $M$ and $M^{\prime}$ are said to be of the same homotopy type if and only if there exist continuous mappings $f: M \rightarrow M^{\prime}, g: M^{\prime} \rightarrow M$, such that $f \circ g$ and $g \circ f$ are homotopic to the identity). 
For example, $L(5,1)$ and $L(5,2)$ have different homotopy types, whereas $L(7,1)$ and $L(7,2)$ have the same homotopy type, but they are not homeomorphic.

A triangulation of $L(p, q)$ is obtained by gluing together $p$ tetrahedra $\left(a_{i}, b_{i}, c_{i}, d_{i}\right), i=$ $0, \ldots, p-1$ according to the following identification of faces $(i+1$ and $i+q$ are understood modulo $p)$ :

$$
\begin{aligned}
& \left(a_{i}, b_{i}, d_{i}\right)=\left(a_{i+1}, b_{i+1}, c_{i+1}\right) \\
& \left(a_{i}, c_{i}, d_{i}\right)=\left(b_{i+q}, c_{i+q}, d_{i+q}\right)
\end{aligned}
$$

The identifications of (3.3) can be realized by embedding the $p$ tetrahedra in euclidean 3space, leading to a "prismatic solid" with $p+2$ vertices $a, b, c_{i}, 2 p$ external faces, $3 p$ external edges, and one internal axis $(a, b)$. Then formula (3.4) is interpreted as the identification of the surface triangles $\left(a, c_{i}, c_{i+1}\right)$ and $\left(b, c_{i+q}, c_{i+1+q}\right)$.

A state on this triangulation made of $p$ tetrahedra $\left(a, b, c_{i}, c_{i+1}\right), i \in \mathbb{Z}_{p}$ is defined by $g=g_{a b}, h_{i}=g_{b c_{i}}, k_{i}=g_{c_{i} c_{i+1}}$. The flatness conditions read:

$$
\begin{gathered}
h_{i+1}=h_{i} k_{i} \\
g_{a c_{i}}=g h_{i}
\end{gathered}
$$

Then identifying faces $\left(a, c_{i}, c_{i+1}\right)$ and $\left(b, c_{i+q}, c_{i+1+q}\right)$ gives

$$
\begin{gathered}
k_{i}=k_{i+q} \\
g h_{i}=h_{i+q}
\end{gathered}
$$

Since $(p, q)=1, k_{i}=k$ is independent of $i,(3.8)$ implies that $g^{p}=e$ and for any $n$, $g^{n} h_{i}=h_{i+n q}$, so that if $n$ is the inverse of $q \bmod p$, one gets $h_{i+1}=g^{n} h_{i}$, which compared to (3.5) gives $k=h_{i}^{-1} g^{n} h_{i}$. Conversely, the data $g, h \in G$ with $g^{p}=e$ determines a state on the above triangulation of $L(p, q)$ through the formulas:

$$
g_{i}=g_{a b}=g, h_{i}=g^{i n} h, k_{i}=h^{-1} g^{n} h .
$$

The weight associated to such a state is:

$$
W(\rho)=\prod_{i=0}^{p-1} \omega\left(g_{i}, h_{i}, k_{i}\right)=\prod_{i=0}^{p-1} \omega\left(g, g^{i n} h, h^{-1} g^{n} h\right)=\prod_{j=0}^{p-1} \omega\left(g, g^{j} h, h^{-1} g^{n} h\right) .
$$

In this case all gauge transformations are of the form:

$$
g \rightarrow \Omega_{a} g \Omega_{a}^{-1}, h \rightarrow \Omega_{a} h \Omega_{c}^{-1}
$$


Gauge invariance means here that for any $g\left(g^{p}=e\right)$ and $h$ :

$$
\prod_{j=0}^{p-1} \omega\left(g, g^{j} h, h^{-1} g^{n} h\right)=\prod_{j=0}^{p-1} \omega\left(g_{A}, g_{A}^{j}, g_{A}^{n}\right)
$$

where $g_{A}$ is a representative of the conjugacy class $C_{A}$ of $g$, an identity which one can prove directly, see formulas (A.2) and (A.3) of the appendix. The Dijkgraaf-Witten prescription to consider a sum over orbits under gauge transformations with, say, $\Omega_{a}=e$, therefore amounts to a sum over $g$ only, leading to the following expression of the invariant of $L(p, q)$ :

$$
Z(L(p, q)) / Z\left(S^{3}\right)=\sum_{g \in G \mid g^{p}=e} \prod_{j=1}^{p-1} \omega\left(g, g^{j}, g^{n}\right)=\sum_{A \mid g_{A}^{p}=e}\left|C_{A}\right| \prod_{j=1}^{p-1} \omega\left(g_{A}, g_{A}^{j}, g_{A}^{n}\right) .
$$

Using (A.4) we arrive at the final expression valid for any 3-cocycle $\omega$ and any finite group:

$$
Z(L(p, q))=|G|^{-1} \sum_{A \mid g_{A}^{p}=e}\left|C_{A}\right| \omega_{A}^{n},
$$

where $\omega_{A}=\prod_{j=1}^{p-1} \omega\left(g_{A}, g_{A}^{j}, g_{A}\right)$ is a $p$-th root of unity built from values of $\omega$ on the cyclic group generated by $g_{A}$ and $n$ is the inverse of $q \bmod p$. Note that if in (2.2) the summation had been taken over the orbits of all gauge tranformations, instead of over the orbits of $\mathcal{G}_{0}$, one would have obtained the different number $|G|^{-1} \sum_{A / g_{A}^{p}=e} \omega_{A}^{n}$, which may not have the right properties, since the so-called global gauge transformations would not have been treated in an appropriate way.

Now we would like to see how these invariants behave with respect to the above classification theorems (3.1), (3.2). From (3.14) we get immediately

$$
Z(L(p, p-q))=Z(L(p, q))^{*}
$$

so that changing the orientation results in complex conjugation.

Next, suppose that $q$ and $q^{\prime}$ are invertible $\bmod p$ and that their product is a square $q q^{\prime}=a^{2} \quad(\bmod p)$, denote by $c$ its inverse and by $b=a c$ the inverse of $a, c=b^{2}$. Let $n, n^{\prime}$ be the inverses of $q, q^{\prime}$, and $\mathcal{C}_{p}=\left\{A \mid g_{A}^{p}=e\right\}$. Since $q b$ is invertible $g \mapsto g^{q b}$ induces a one-to-one mapping of $\mathcal{C}_{p}$ into itself: $A \mapsto B$ such that $g_{B}$ is conjugate to $g_{A}^{q b}$. Furthermore $\left|C_{A}\right|=\left|C_{B}\right|$, so that using (A.4) we can rewrite (3.13) as:

$$
\begin{aligned}
& Z(L(p, q)) / Z\left(S^{3}\right)=\sum_{B \in \mathcal{C}_{p}}\left|C_{B}\right| \prod_{j=1}^{p-1} \omega\left(g_{B}, g_{B}^{j}, g_{B}^{n}\right)=\sum_{A \in \mathcal{C}_{p}}\left|C_{A}\right| \prod_{j=1}^{p-1} \omega\left(g_{A}^{q b}, g_{A}^{j q b}, g_{A}^{q b n}\right) \\
& \quad=\sum\left|C_{A}\right| \prod_{j^{\prime}=1}^{p-1} \omega\left(g_{A}^{q b}, g_{A}^{j^{\prime}}, g_{A}^{b}\right)=\sum\left|C_{A}\right| \omega_{A}^{q c}=\sum_{A \in \mathcal{C}_{p}}\left|C_{A}\right| \omega_{A}^{n^{\prime}}=Z\left(L\left(p, q^{\prime}\right)\right) / Z\left(S^{3}\right) .
\end{aligned}
$$


We have therefore directly shown that for any 3-cocycle $\omega$ and any finite group, $Z(M)$, in the class of lens spaces, is an invariant which is sensitive to orientation through complex conjugation, and takes the same value for all manifolds of the same (oriented) homotopy type. We do not know if this holds in general.

Let us now specialize to the case of a cyclic group $G=\mathbb{Z}_{N}$ whose cohomology group $H^{3}(G, U(1))$ is cyclic of order $N$, with representative cocycles:

$$
\omega_{N}^{l}(x, y, z)=\exp \left(\frac{2 i \pi l}{N^{2}} \bar{z}(\bar{x}+\bar{y}-\overline{x+y})\right)
$$

where $l=0, \ldots, N-1$, and $\bar{x}$ denotes the integer between 0 and $N-1$ representing an element $x \in \mathbb{Z}_{N}$. $\omega_{N}^{l}$ is equal to 1 except if $\bar{x}+\bar{y} \geq N$ when its value is $\exp (2 i \pi l \bar{z} / N)$. Put $m=(N, p)$. We shall now prove that in this case (3.14) is equal to:

$$
\frac{Z(L(p, q))}{Z\left(S^{3}\right)}=\sum_{k=0}^{m-1} \exp \left(\frac{2 i \pi l p n}{m^{2}} k^{2}\right)
$$

where $n$ is the representative in $\{0, \ldots, p-1\}$ of the inverse of $q \bmod p$. If $m=1$ this invariant equals 1 , otherwise it equals the value of a Gauss sum $S(a, m)$ with $a=\ln p / m$ :

$$
Z(L(p, q)) / Z\left(S^{3}\right)=S(a, m)=\sum_{k=0}^{m-1} \exp \left(2 i \pi a k^{2} / m\right) .
$$

To prove (3.18) we translate the multiplicative notations for the group $G$, taken to be the group of $N$-th roots of 1 , into additive ones by the formula

$$
g=\exp (2 i \pi \bar{g} / N), \bar{g} \in\{0, \ldots, N-1\}
$$

Then

$$
g^{p}=e \Leftrightarrow N\left|p \bar{g} \Leftrightarrow \frac{N}{m}\right| \frac{p}{m} \bar{g} \Leftrightarrow \exists k \in\{0, \ldots, m-1\}, \quad \bar{g}=k N / m
$$

and (3.14) reduces to:

$$
Z(L(p, q)) / Z\left(S^{3}\right)=\sum_{k=0}^{m-1} \exp \left(2 i \pi \ln k\left|\mathcal{S}_{k}\right| / m\right)
$$

where $\left|\mathcal{S}_{k}\right|$ is the number of elements of the set:

$$
\mathcal{S}_{k}=\{j \in\{1, \ldots, p-1\} \mid \bar{g}+\overline{j \bar{g}} \geq N\}
$$

(Remember that from (3.20), $\bar{g}$ depends on $k$.) But since $\bar{g}(j+m /(m, k))=\bar{g} j+N k /(m, k)$, one has

$$
\left|\mathcal{S}_{k}\right|=\frac{p}{m}(m, k)\left|\left\{j \in\left\{1, \ldots, \frac{m}{(m, k)}-1\right\} \mid \bar{g}+\overline{j \bar{g}} \geq N\right\}\right| .
$$


Writing

$$
j \bar{g}=j \frac{k}{(m, k)} \frac{(m, k) N}{m}
$$

and noticing that $j \mapsto j^{\prime}=j k /(m, k)$ is a one-to-one map of $\mathbb{Z}_{m /(m, k)}$ into itself, we get:

$$
\left|\mathcal{S}_{k}\right|=\frac{p}{m}(m, k)\left|\left\{j^{\prime} \in\left\{1, \ldots, \frac{m}{(m, k)}-1\right\} \mid \bar{g}+\overline{j^{\prime}(m, k) N / m} \geq N\right\}\right| .
$$

Now set $J=(m /(m, k))-j^{\prime}$. From $\overline{j^{\prime}(m, k) N / m}=N-J(m, k) N / m$, one finds $\bar{g}+\overline{j^{\prime}(m, k) N / m} \geq N \Leftrightarrow J \in\{1, \ldots k /(m, k)\}$, leading finally to $\left|\mathcal{S}_{k}\right|=p k / m$ and to (3.18).

\section{Surgery invariants}

All lens spaces $L(p, q), 0<q<p,(p, q)=1$ are known [14] to have a surgery presentation given by a finite chain of $n$ unknotted circles with integer surgery coefficients $p_{1}, p_{2}, \ldots, p_{n}$, all $\geq 2$. Such a chain represents the space $L\left(A_{n}, A_{n-1}\right)$ where $A_{n}$ is defined recursively by:

$$
\begin{gathered}
A_{0}=1, A_{1}=p_{1}, A_{2}=p_{1} p_{2}-1, A_{3}=p_{1} p_{2} p_{3}-p_{1}-p_{3} \\
A_{n}\left(p_{1}, \ldots, p_{n}\right)=p_{n} A_{n-1}\left(p_{1}, \ldots, p_{n-1}\right)-A_{n-2}\left(p_{1}, \ldots, p_{n-2}\right) .
\end{gathered}
$$

The last relation is equivalent to $A_{n} / A_{n-1}=p_{n}-A_{n-2} / A_{n-1}$, leading to the finite continued fraction:

$$
\frac{A_{n}\left(p_{1}, \ldots, p_{n}\right)}{A_{n-1}\left(p_{1}, \ldots, p_{n-1}\right)}=p_{n}-\frac{1}{\ldots-\frac{1}{p_{2}-\frac{1}{p_{1}}}}
$$

Note that since $A_{n}-A_{n-1}=\left(p_{n}-1\right) A_{n-1}-A_{n-2}$, and $p_{1}, p_{2}, \ldots, p_{n} \geq 2$ then

$$
A_{n}>A_{n-1}>\ldots>A_{1}>A_{0}=1 \text {. }
$$

One also has

$$
A_{n-1}\left(p_{1}, \ldots, p_{n-1}\right) A_{n-1}\left(p_{2}, \ldots, p_{n}\right)-A_{n}\left(p_{1}, \ldots, p_{n}\right) A_{n-2}\left(p_{2}, \ldots, p_{n-1}\right)=1
$$

i.e. $q=A_{n-1}\left(p_{1}, \ldots, p_{n-1}\right)$ is prime to $p=A_{n}\left(p_{1}, \ldots, p_{n}\right)$ and the inverse of $q \bmod p$ is $q^{\prime}=A_{n-1}\left(p_{2}, \ldots, p_{n}\right)$. The proof of (4.5) goes by induction. Conversely, any non-negative rational number $p / q$ greater than 1 can be expressed as a fraction like (4.3) with all $p_{i}$ 's greater or equal than 2 : set $B_{0}=p, B_{1}=q$ and since $(p, q)=1$ one can define recursively strictly decreasing integers $B_{k}>0$ and integers $q_{k} \geq 2$ such that $B_{k-1}=q_{k} B_{k}-B_{k+1}$. Stop at step $n$ when $B_{n}=1$ and $q_{n}=B_{n-1}$. Then

$$
\frac{p}{q}=\frac{B_{0}}{B_{1}}=q_{1}-\frac{B_{2}}{B_{1}}=q_{1}-1 /\left(q_{2}-\frac{B_{3}}{B_{2}}\right)=\cdots
$$


giving a fraction like $(4.3)$ with $\left(p_{1}, \ldots, p_{n}\right)=\left(q_{n}, \ldots, q_{1}\right)$.

From the data $G, \omega$ one defines [9] the finite dimensional algebra $D^{\omega}(G)$ over $\mathbb{C}$ in terms of a basis $\left\{g \underset{x}{L_{f}}\right\}, g, x \in G$ as follows:

$$
g L_{x} h \bigsqcup_{y}=\delta_{g, x h x^{-1}} \theta_{g}(x, y){ }_{g y}
$$

where

$$
\theta_{g}(x, y)=\omega(g, x, y) \omega\left(x, y,(x y)^{-1} g x y\right) \omega\left(x, x^{-1} g x, y\right)^{-1} .
$$

This algebra is associative by virtue of (A.1) and has unit element $1=\sum_{g \in G} g\llcorner$. It has the structure of a ribbon quasi-Hopf quasitriangular algebra, described in detail in $[9,4]$ allowing one to define topological invariants of 3-manifolds $\mathcal{F}(M)$ by the method explained in [4] and based on the construction of manifolds by surgery. By induction on $n$, the number of circles defining the lens space by surgery, we find:

$$
\begin{gathered}
\mathcal{F}\left(L\left(A_{n}, A_{n-1}\right)\right)=\sum_{g \mid g^{A_{n}}=e} \prod_{k=0}^{n-2} \theta_{g^{P_{k}}}\left(g^{P_{k+1}}, g^{-P_{k+1}}\right) \prod_{l=1}^{n-1} \theta_{g^{P_{l}}}\left(g^{p_{l+1} P_{l}}, g^{P_{l-1}}\right) \\
\prod_{m=1}^{n} \prod_{j_{m}=1}^{p_{m}-1} \omega\left(g^{P_{m-1}}, g^{j_{m} P_{m-1}}, g^{P_{m-1}}\right)
\end{gathered}
$$

where $P_{k}=(-1)^{k} A_{k}\left(p_{1}, \ldots, p_{k}\right)$ and $A_{k}$ is given by (4.2). As a consequence of our conjecture, this expression must be equal to (3.14). We have checked this equality with a computer for $G=\mathbb{Z}_{N}, \omega=\omega_{N}^{l}$. It is clear that a proof of this equality would already rely on a consequent amount of identities such as those given in appendix. So far we have only been able to prove it for any $G, \omega$ in the cases $n=1$, where (4.8) reduces to (3.13), and $n=2,\left(p_{1}, p_{2}\right)=1$.

Acknowledgements: A.C. thanks LAPP for the kind hospitality and friendly atmosphere.

\section{A Appendix}

We give here some identities involving normalized cocycles which we found rather useful. The first one establishes that (4.7) defines a "twisted 2-cocycle", i.e. :

$$
\theta_{g}(x, y) \theta_{g}(x y, z)=\theta_{g}(x, y z) \theta_{x^{-1} g x}(y, z)
$$

To prove it, simplify successively (A.1) with the help of (1.1) written with $\left(g_{i}\right)$ replaced by $\left(x, y, z,(x y z)^{-1} g x y z\right),\left(x, y,(x y)^{-1} g x y, z\right),(g, x, y, z)$ and finally $\left(x, x^{-1} g x, y, z\right)$.

In the following, we suppose that $p$ is a positive integer such that $g^{p}=e$, but $h$ can be any element of $G$. Then the results which are used in the main text are

$$
\prod_{j=0}^{p-1} \omega\left(g, g^{j} h, h^{-1} g^{n} h\right)=\prod_{j=0}^{p-1} \omega\left(g, g^{j}, g^{n}\right)
$$




$$
\begin{gathered}
\prod_{j=0}^{p-1} \omega\left(g, g^{j}, g\right)=\prod_{j=0}^{p-1} \omega\left(h^{-1} g h, h^{-1} g^{j} h, h^{-1} g h\right) \\
\prod_{j=0}^{p-1} \omega\left(g^{m}, g^{j}, g^{n}\right)=\left(\omega_{g}\right)^{m n}
\end{gathered}
$$

where $\omega_{g}$ is a $p$-th root of 1 . Together (A.3) and (A.4) imply that $\omega_{g}=\omega_{A}$ only depends on the conjugacy class $C_{A}$ of $g$. To prove (A.2), we form the product of $k$ copies of the cocycle condition (1.1), substituting $\left(g_{i}\right)$ with $\left(g, g^{j}, g^{n}, h\right), j=0, \ldots, k-1$, and divide it by a similar product for $\left(g_{i}\right)=\left(g, g^{j}, h, h^{-1} g^{n} h\right)$. We obtain

$$
\prod_{j=0}^{k-1} \frac{\omega\left(g, g^{j}, g^{n}\right)}{\omega\left(g, g^{j} h, h^{-1} g^{n} h\right)} \frac{\prod_{j=0}^{k+n-1} \omega\left(g, g^{j}, h\right)}{\prod_{j=0}^{k-1} \omega\left(g, g^{j}, h\right) \prod_{j=0}^{n-1} \omega\left(g, g^{j}, h\right)}=\frac{\omega\left(g^{k}, g^{n}, h\right)}{\omega\left(g^{k}, h, h^{-1} g^{n} h\right)} .
$$

Setting $k=p$ we get (A.2). Another useful formula is the case $n=1$ of (A.5):

$$
\prod_{j=0}^{k-1} \omega\left(g, g^{j} h, h^{-1} g h\right)=\theta_{g}\left(g^{k}, h\right) \prod_{j=0}^{k-1} \omega\left(g, g^{j}, g\right) .
$$

To prove (A.3), form the products of $k$ cocycle relations for $\left(g_{i}\right)$ replaced by $\left(g, h, h^{-1} g^{j} h, h^{-1} g h\right),\left(h^{-1}, g h, h^{-1} g^{j} h, h^{-1} g h\right),\left(h^{-1}, h, h^{-1} g^{j} h, h^{-1} g h\right)$, then simplify a factor involving five $\omega^{\prime}$ 's using the cocycle relations written with $\left(h^{-1}, h, h^{-1} g^{k} h, h^{-1} g h\right)$ and $\left(h^{-1}, h, h^{-1} g h, h^{-1} g^{k} h\right)$. This gives

$$
\prod_{j=0}^{k-1} \omega\left(g, g^{j} h, h^{-1} g h\right)=\theta_{g}\left(h, h^{-1} g^{k} h\right) \prod_{j=0}^{k-1} \omega\left(h^{-1} g h, h^{-1} g^{j} h, h^{-1} g h\right),
$$

thus setting $k=p$ and using (A.2) leads to (A.3). To prove (A.4) consider the product of $p$ cocycle relations for $\left(g_{i}\right)=\left(g^{m}, g^{j}, g^{n_{1}}, g^{n_{2}}\right)$. It is then easy to see that $n \mapsto \prod_{j} \omega\left(g^{m}, g^{j}, g^{n}\right)$ is a homomorphism from the additive group $\mathbb{Z}_{p}$ into $U(1)$. Therefore this product is of the form:

$$
\prod_{j=0}^{p-1} \omega\left(g^{m}, g^{j}, g^{n}\right)=\exp (2 i \pi \bar{\omega}(m) n / p) .
$$

By a similar argument with $\left(g_{i}\right)=\left(g^{m_{1}}, g^{m_{2}}, g^{j}, g\right)$ one sees that $\bar{\omega}(m)$ is a homomorphism from $\mathbb{Z}_{p}$ to $\mathbb{Z}_{p}$, thereby proving (A.4).

Notice that (A.5),(A.6),(A.7) are valid without any assumption on $g, h \in G$.

There are many other identities involving products one can derive. For example, for any positive integers $k, l$ and any $g \in G$ :

$$
\prod_{j=0}^{k+l-1} \omega\left(g, g^{j}, g\right)=\theta_{g}\left(g^{l}, g^{k}\right) \prod_{j=0}^{k-1} \omega\left(g, g^{j}, g\right) \prod_{j=0}^{l-1} \omega\left(g, g^{j}, g\right)
$$


and this implies the symmetry $\theta_{g}\left(g^{k}, g^{l}\right)=\theta_{g}\left(g^{l}, g^{k}\right)$. This can be proved as follows: set $Q_{k}=\theta_{g}\left(g^{l}, g^{k}\right) / \prod_{j=k}^{k+l-1} \omega\left(g, g^{j}, g\right)$. The cocycle relation with $\left(g_{i}\right)=\left(g, g^{l}, g^{k}, g\right),\left(g^{l}, g, g^{k}, g\right)$ implies that $Q_{k+1}=Q_{k}$, which is therefore equal to $Q_{0}$. There is a similar identity with negative powers:

$$
\prod_{j=0}^{k+l-1} \omega\left(g, g^{-j}, g\right)=\frac{\omega\left(g, g^{-l}, g\right)}{\theta_{g}\left(g^{-l}, g^{1-k}\right)} \prod_{j=0}^{k-1} \omega\left(g, g^{-j}, g\right) \prod_{j=0}^{l-1} \omega\left(g, g^{-j}, g\right)
$$

and relations between products with positive and negative powers:

$$
\begin{gathered}
\prod_{j=0}^{l} \omega\left(g, g^{-j}, g\right)=\theta_{g}\left(g^{-l}, g^{l}\right) \prod_{j=0}^{l-1} \omega\left(g, g^{j}, g\right) \\
\prod_{j=0}^{k-1} \omega\left(g^{-1}, g^{-j}, g^{-1}\right)=\theta_{g}\left(g^{-k}, g^{k}\right) \theta_{g^{-k}}\left(g^{-1}, g\right) \prod_{j=0}^{k-1} \omega\left(g, g^{j}, g\right) .
\end{gathered}
$$

The proof of (A.9) is by setting $R_{k}=\theta_{g}\left(g^{-l}, g^{1-k}\right) \prod_{j=k}^{k+l-1} \omega\left(g, g^{-j}, g\right)$; then (1.1) with $\left(g_{i}\right)=$ $\left(g^{-l}, g, g^{-k}, g\right),\left(g, g^{-l}, g^{-k}, g\right)$ implies $R_{k+1}=R_{k}$, which is therefore equal to $R_{1}$. The proof of (A.10) is by induction and use of (1.1) with $\left(g_{i}\right)=\left(g, g^{-l-1}, g^{l+1}, g\right),\left(g, g^{-l-1}, g, g^{l+1}\right)$, $\left(g^{-l}, g, g^{l}, g\right),\left(g, g^{-l}, g^{l}, g\right)$. The proof of (A.11) is again by induction and use of (1.1) with $\left(g_{i}\right)=\left(g^{-k-1}, g, g^{k}, g\right),\left(g, g^{-k-1}, g, g^{k}\right),\left(g^{-1}, g^{-k}, g^{-1}, g\right),\left(g^{-1}, g, g^{-k-1}, g\right)$.

Note that replacing $g$ by $g^{-1}$ in (A.11) and substituting the result in the original identity we get:

$$
\theta_{g}\left(g^{-k}, g^{k}\right) \theta_{g^{-k}}\left(g^{-1}, g\right) \theta_{g^{-1}}\left(g^{k}, g^{-k}\right) \theta_{g^{k}}\left(g, g^{-1}\right)=1
$$

\section{References}

[1] R. Dijkgraaf and E. Witten, Commun. Math. Phys. 129 (1990) 393.

[2] E. Witten, Commun. Math. Phys. 121 (1989) 351.

[3] R. Dijkgraaf, C. Vafa, E. Verlinde and H. Verlinde, Commun. Math. Phys. 123 (1989) 485.

[4] D. Altschuler and A. Coste, Quasi-Quantum Groups, Knots, 3-Manifolds, and Topological Field Theory, preprint CERN-TH 6360/92, to appear in Commun. Math. Phys.

[5] N. Reshetikhin and V. G. Turaev, Commun. Math. Phys. 127 (1990) 1.

[6] N. Reshetikhin and V.G. Turaev, Inv. Math. 103 (1991) 547. 
[7] V. G. Drinfeld, Quasi-Hopf algebras and the Knizhnik-Zamolodchikov equations, in Problems of modern quantum field theory, ed. A. Belavin et al. (Springer, 1990).

V. G. Drinfeld, Leningrad Math. J. 1 (1991) 1419.

[8] R. Kirby, Inv. Math. 45 (1978) 35.

R. Fenn and C. Rourke, Topology 18 (1978) 1.

[9] R. Dijkgraaf, V. Pasquier and P. Roche, in proc. workshop Integrable Systems and Quantum Groups, Pavia, 1990; and in proc. Int. coll. on Modern Quantum Field Theory, Tata Institute, Bombay, 1990.

[10] G. Felder, private communication.

O. Grandjean, diploma thesis, ETH Zürich (1992).

[11] D. Freed and F. Quinn, Chern-Simons theory with finite gauge groups, preprint, 1991.

[12] N. Steenrod, The topology of fiber bundles (Princeton University press, 1951).

[13] M. Hennings, Math. Proc. Camb. Phil. Soc. 109 (1991) 59.

[14] D. Rolfsen, Knots and Links (Publish or Perish, Berkeley, 1976). 